\title{
Las expectativas de autoeficacia y el ajuste emocional en el afrontamiento de la fibromialgia
}

\author{
Miguel Ángel Vallejo Pareja, $\mathrm{M}^{\mathrm{a}}$ Isabel Comeche Moreno, José Ortega Pardo, \\ $\mathrm{M}^{\mathrm{a}}$ de la Fe Rodríguez Muñoz y Marta I. Díaz García
}

Universidad Nacional de Educación a Distancia (UNED)

Disponible online 30 de abril de 2009

\begin{abstract}
El objetivo fue evaluar de forma descriptiva la relación entre las expectativas de autoeficacia, malestar emocional y estrategias de afrontamiento en pacientes con fibromialgia, considerando los componentes físicos y psicosociales de la experiencia de dolor, y sobre todo la necesidad de clarificar la relación entre ellos para mejorar la adaptación a cualquier situación de dolor crónico. En el estudio participaron 100 mujeres pacientes de fibromialgia del Instituto Provincial de Rehabilitación del Hospital Universitario Gregorio Marañón de Madrid quienes completaron la siguiente batería de cuestionarios: la Escala de ansiedad y depresión en hospital (HADS), el Inventario de estrategias de afrontamiento en dolor (CPCI), y la Escala de autoeficacia en dolor crónico (CPSS). Los resultados muestran que la autoeficacia correlaciona inversamente con el malestar emocional (ansiedad y depresión). Con relación a las estrategias de afrontamiento, la persistencia en la tarea se relaciona significativamente con las expectativas de autoeficacia, mientras que esta variable lo hace de forma inversa con la depresión. Descansar y protegerse se relacionan de forma negativa con la autoeficacia. Estos resultados sugieren que las expectativas de autoeficacia juegan un papel importante en el uso de estrategias positivas de afrontamiento y en el funcionamiento emocional adecuado en pacientes con fibromialgia.
\end{abstract}

Palabras clave:

Fibromialgia, autoeficacia, ansiedad, depresión, afrontamiento.

The objective was to evaluate the relationship between of self-efficacy expectations, psychological distress and coping strategies among subjects with fibromyalgia, given the physical and psychosocial components of pain, and specially the need to clarify the relationship between them in order to improve the adjustment to any chronic pain condition. One-hundred women diagnosed of fibromyalgia from the rehabilitation center at the Hospital Universitario Gregorio Marañón de Madrid completed the following set of questionnaires, the Hospital Anxiety and Depression Scale (HADS), the Chronic Pain Coping Inventory (CPCI) and the Chronic Pain Self-efficacy Scale (CPSS). The results showed that self-efficacy was inversely correlated with psychological distress (anxiety and depression). Respect to coping strategies, task persistence was significantly related to expectations of self-efficacy, while this variable was inversely correlated with depression. A negative relation was found between guarding, resting and self-efficacy. These findings suggest that expectations of self-efficacy may have greater explanatory power over the use of positive coping strategies and correct emotional functioning in FMS patients.

Keywords:

Fibromyalgia, self-efficacy, anxiety, depression, coping.

Correspondencia: Miguel Ángel Vallejo Pareja. Departamento de Personalidad, Evaluación y Tratamiento Psicológicos, Facultad de Psicología, Universidad Nacional de Educación a Distancia (UNED). C/ Juan del Rosal, 10.28040 Madrid (Spain). E-mail: mvallejo@psi.uned.es 
La fibromialgia es un síndrome reumatológico en el que aparece dolor crónico generalizado de tipo musculoesquelético (Bayazit, Gürsoy, Özer, Karakurum y Madenci, 2002). En la fibromialgia, como en otros trastornos de dolor crónico, no sólo existe la presencia prolongada de estimulación nociceptiva, sino también aspectos emocionales, conductuales y cognitivos interrelacionados que determinan la experiencia de dolor (Bettina y cols., 2008; Meeus, 2007).

De las diferentes variables que influyen en la experiencia de dolor crónico, dentro de las variables psicológicas y más concretamente de las cognitivas, las expectativas de autoeficacia han demostrado cumplir un papel importante. Partiendo de la teoría del aprendizaje social propuesta por Bandura (1977) se entiende que considerarse con capacidad para realizar diferentes actividades influye en la utilización de estrategias de afrontamiento y en la respuesta emocional frente al dolor. De hecho, se ha señalado que, en pacientes con dolor crónico, las expectativas de autoeficacia pueden llegar a tener una capacidad predictiva sobre el funcionamiento físico mayor que la propia intensidad de dolor (Arnstein, 2000; Meredith, Strong y Feeney, 2006). En esta misma línea, en la que se destaca la importancia de las expectativas de autoeficacia, Buckelew et al. (1994) concluyeron que dichas expectativas tenían un valor predictivo sobre la manifestación de conductas de dolor mayor que la depresión. En otros trabajos se ha puesto de manifiesto que la autoeficacia ha resultado ser un determinante significativo del funcionamiento físico en pacientes con fibromialgia (Buckelew, Murria, Hewett, Jonson y Huyser, 1995), además de un buen predictor de los cambios una vez finalizado un programa de ejercicio y del mantenimiento de los logros y mejoras a largo plazo (Buckelew et al., 1996; Oliver y Cronan, 2005).

En términos generales, la bibliografía ha destacado el papel de las estrategias de afrontamiento como mecanismo de adaptación, manejo y control del estrés producido por el dolor crónico. Aunque es necesario señalar que no todas las estrategias funcionan de la misma manera, siendo las estrategias activas y las centradas en el problema las más eficaces para manejar el dolor mientras que la distracción y el catastrofismo parecen estar relacionadas con un aumento del dolor crónico (Rodríguez-Parra, Esteve y López, 2000).

Respecto a la relación entre las expectativas de autoeficacia y el empleo de estrategias de afrontamiento, Jensen, Turner y Romano (1991) encontraron un nexo consistente entre los esfuerzos de afrontamiento informados por los pacientes y las creencias de los mismos en su capacidad para utilizar estrategias como el ejercicio activo, el reposo, la ignorancia del dolor o la toma de medicación. Además, las expectativas de autoeficacia no sólo parecen incrementar los comportamientos emprendidos para enfrentarse al dolor sino que podrían influir en el tipo de estrategia utilizada (Asghari y Nicholas, 2001; Brister, Turner Aaron y Mancl, 2006; Buescher et al., 1991). En este sentido, los trabajos anteriores concluyeron que los pacientes de dolor crónico con un alto nivel de autoeficacia parecen conocer y confiar más en estrategias activas como persistencia en la tarea, en detrimento de las pasivas, como descansar. Sin embargo, al caracterizarse este síndrome por una mayor percepción de pérdida de control en comparación con otras patologías reumáticas (Buckhard y Bjelle, 1996) y un significativo empleo de estrategias pasivas (Soucase, Monsalve, Soriano y De Andrés, 2004), incluso en mayor medida que otros trastornos de dolor crónico (Mellegard, Grossi, y Soares, 2001), podrían ser necesarios más estudios que tuviesen en cuenta otras estrategias de afrontamiento con el fin de ampliar y / o reafirmar el conocimiento sobre dicha relación con las consiguientes repercusiones clínicas o terapéuticas que ello conllevaría.

Por último, considerando las reacciones emocionales en la fibromialgia, han cobrado especial relevancia los trabajos que se centran en la ansiedad y la depresión (Benett, 2002; PérezPareja et al., 2004) como patologías asociadas más comunes en este trastorno. Con respecto a los factores emocionales y a las variables cognitivas, diversos estudios han encontrado una relación similar a la hallada entre autoeficacia y afrontamiento (Altman, 2004; Arnstein, Caudill, Mandle, Norris y Beasley, 1999; Rahman, Ambler, Underwood y Shipley, 2004). Así, Altman (2004) señaló que junto al catastrofismo, considerarse con capacidad para manejar el dolor, y afrontarlo, sería un mediador significativo de la relación entre malestar emocional y dolor. Específicamente, en el síndrome de fibromialgia, las expectativas de autoeficacia parecen ser uno de los factores que afectan o influyen sobre la depresión (Han y Lee, 2005; Sung, Shin, y Lee, 2003). Sin embargo, teniendo en cuenta tanto la relación positiva entre el malestar emocional y el mantenimiento y agravamiento del dolor (Dresh, Polatin y Gatchel, 2002; Kerns, Roserberg y Jacob, 1994; McCracken, Zayfert y Gross, 1992; Turk, Okifuji, y Scharff, 1995;) y sobre todo la relación diferencial entre ansiedad, depresión y dolor en fibromialgia (Kurtze, Gundersen y Svebak, 1998; Kurtze y Svebak, 2001; Thieme, Turk y Flor, 2004), un mejor conocimiento de la relación entre variables emocionales y expectativas de autoeficacia estaría justificado, dada la influencia que éstas últimas parecen ejercer sobre las respuestas emocionales características del dolor crónico.

Por todo ello, el objetivo de este trabajo es estudiar de forma descriptiva las relaciones entre las expectativas de autoeficacia, las reacciones emocionales (ansiedad y depresión) y las estrategias de afrontamiento en pacientes con fibromialgia.

Método

\section{Participantes}

La muestra estaba compuesta por 100 mujeres con fibromialgia, pacientes del Instituto Provincial de Rehabilitación (IPR) del Hospital Universitario Gregorio Marañón de Madrid, diagnosticadas de fibromialgia de acuerdo a los criterios de cla- 
sificación del American College of Rheumatology (Wolfe et al., 1999). La media de edad fue de 47 años (rango=23-77, desviación estándar=8.26). Al inicio de este estudio exploratorio, que era a su vez parte de un estudio más amplio en el que además se tenían en cuenta variables fisiológicas, las pacientes fueron informadas sobre el tipo de estudio y el carácter confidencial de los datos. Una vez dado su consentimiento, completaban una batería de cuestionarios de forma individual relativos tanto a los datos demográficos más relevantes (edad, sexo, profesión, años con el dolor y años con el diagnóstico de fibromialgia) como a las variables psicológicas de interés: factores emocionales (ansiedad y depresión), factores conductuales (estrategias de afrontamiento) y factores cognitivos (autoeficacia percibida). El estudio fue realizado en el primer semestre de 2006.

\section{Instrumentos}

La evaluación de las variables objetivo de este estudio se realizó mediante los siguientes cuestionarios:

Escala de ansiedad y depresión en hospital (HADS) (Zigmond y Snaith, 1983). La escala HADS es una escala autoaplicada que consta de 14 ítems. Estos ítems se dividen en dos subescalas de 7, evaluando ansiedad y depresión cada una de las dimensiones. El rango de respuesta oscila entre 0 y 21. Esta puntuación se obtiene al sumar el valor de cada ítem. Zigmond y Snaith (1994) recomiendan que se interpreten los resultados de las dos subescalas por separado. Un resultado entre 8 y 10 indica casos leves, entre 11 y 15 casos moderados y 16 o más indica casos severos (Cooper-Evans, Alderman, Kinht y Oddy, 2008).

Inventario de estrategias de afrontamiento del dolor crónico (CPCI) (Jensen, Turner, Romano y Strom, 1995). Este es un cuestionario que consta de 64 ítems, evalúa las estrategias utilizadas por los pacientes a la hora de manejar el dolor, agrupándolas en 8 categorías diferentes: protegerse, descansar, buscar ayuda, relajarse, continuar con la tarea, ejercicio físico, buscar apoyo social y auto-instrucciones de afrontamiento. La puntuación para cada una de las dimensiones está comprendida entre 0 y 7 ya que representa el número de días que durante la semana pasada el paciente utilizó cada una de las estrategias específicas para afrontar el dolor.

Escala de autoeficacia de dolor crónico (CPSS) (Anderson, Dowds, Pelletz, Edwards, y Peeters, 1995). El CPSS es una versión modificada a partir de la escala de Autoeficacia para Artritis (Lorig, Chastain, Ung, Shoor y Holman, 1989). Está formada por 22 ítems agrupados a su vez en 3 subescalas: autoeficacia para el manejo del dolor, autoeficacia en las actividades físicas y autoeficacia en el manejo de los síntomas. El formato de respuesta es tipo likert con un rango comprendido entre 0 (nada seguro) y 10 (totalmente seguro).
Resultados

En primer lugar, y en relación con las variables emocionales evaluadas, señalar que la puntuación media de la muestra en la subescala de depresión del HAD es de 7.80 ( $D T=4.05)$, puntuación que se encuentra en el intervalo de depresión dudosa o subclínica. Por otra parte, la puntuación media de la muestra en esta subescala de ansiedad es de $9.80(D T=4.7)$, puntuación que también se encuentra en el intervalo de ansiedad subclínica.

Como puede verse en la Figura 1, la distribución de la muestra que un $25.20 \%$ de los pacientes tiene puntuaciones de depresión como problema clínico, un $27.20 \%$ un nivel de depresión subclínica y el resto (47.60\%) muestra niveles normales de depresión. Con respecto a la distribución de las puntuaciones de ansiedad, un $44 \%$ de los pacientes manifiestan ansiedad como problema clínico, un $24 \%$ tiene un nivel de ansiedad subclínica y el resto (32\%) muestra niveles normales de ansiedad.

Figura 1. Porcentaje de ansiedad y depresión de la muestra.

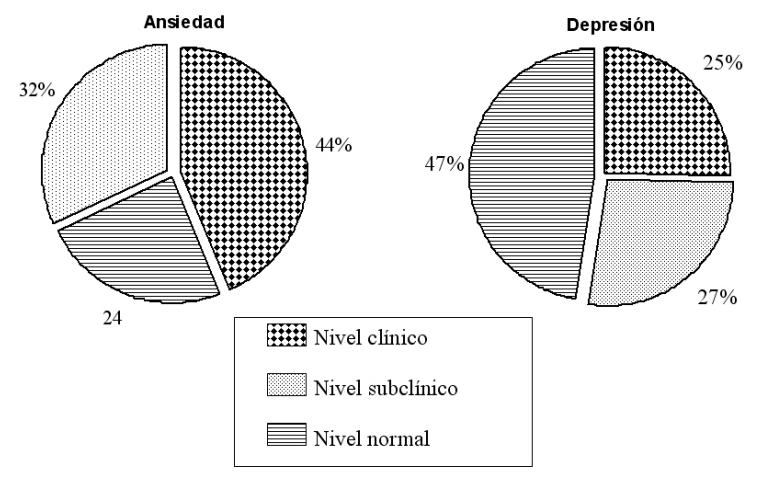

En segundo lugar, y considerado las estrategias de afrontamiento, la persistencia en la tarea $(M=3.67 ; D T=1.29)$, protegerse $(M=3.39 ; D T=1.23)$, descansar $(M=3.38 ; D T=1.27)$ y las auto-instrucciones de afrontamiento $(M=3.11 ; D T=1.30)$ han sido las estrategias más utilizadas por la muestra, siendo la relajación la menos utilizada $(M=1.80, D T=1.23)$ (Tabla 1).

Tabla 1. Estrategias de afrontamiento utilizadas ante el dolor según las subescalas del CPCI.

\begin{tabular}{lcc}
\hline Estrategia de afrontamiento & $\boldsymbol{M}$ (escala $\mathbf{0 - 7})$ & $\boldsymbol{D T}$ \\
\hline Relajación & 1.80 & 1.23 \\
Ejercicio & 2.06 & 1.38 \\
Petición de ayuda & 2.32 & 1.31 \\
Búsqueda de apoyo social & 2.89 & 1.33 \\
Auto-instrucciones de afrontamiento & 3.11 & 1.30 \\
Descansar & 3.38 & 1.27 \\
Protegerse & 3.39 & 1.23 \\
Persistencia en la tarea & 3.67 & 1.29 \\
Afrontamiento global & 2.75 & 1.39 \\
\hline
\end{tabular}


Por último, se realizó un análisis correlacional entre las subescalas del cuestionario de autoeficacia (CPSS), las respuestas emocionales evaluadas en la muestra, ansiedad y depresión respectivamente (HADS), y las estrategias de afrontamiento al dolor (CPCI) más valoradas, y por tanto más utilizadas por la muestra. En la tabla sólo se han señalado aquellas variables que se han asociado de forma significativa $(p<.01)$.

De las tres subescalas que componen el cuestionario de autoeficacia, sólo se han obtenido relaciones significativas inversas entre la autoeficacia global $(r=-.50)$, la referida a actividades físicas $(r=-.48)$ y la autoeficacia en el manejo de los síntomas $(r=-.55)$ y la ansiedad. Es decir, experimentar ansiedad correlaciona negativamente con la realización de actividades físicas y los intentos de manejar las consecuencias de la fibromialgia.

Con respecto a la correlaciones entre depresión y autoeficacia se han encontrado correlaciones negativas entre estas variables (depresión y autoeficacia global, $r=-.49$; depresión y manejo del dolor, $r=-.28$; depresión y actividades físicas $r=-.38$ y depresión y manejo de los síntomas $r=-.49$ ). Estos datos apuntan hacia una relación entre las estrategias de autoeficacia y el padecimiento de síntomas depresivos bastante parecida a la relación que se acaba de comentar entre dichas estrategias y la ansiedad. Es decir, las puntuaciones elevadas en depresión se asocian con un peor manejo de las actividades físicas y de los síntomas de la fibromialgia.

Finalmente, señalar que el nivel de correlación entre el padecimiento de ansiedad y depresión es significativo $(r=.65)$, lo que parece indicar una tendencia a que en los pacientes con fibromialgia se presenten a la vez síntomas de los dos trastornos (Tabla 2).

Tabla 2. Correlaciones significativas $(p<.01)$ entre las subescalas de la autoeficacia y las variables emocionales (ansiedad y depresión) y conductuales (estrategias de afrontamiento más utilizadas).

\begin{tabular}{lccccc}
\hline & Ansiedad & Depresión & \multicolumn{2}{c}{ Estrategias de afrontamiento } \\
& & & Evitación & Descanso & $\begin{array}{c}\text { Persisten- } \\
\text { cia }\end{array}$ \\
\hline $\begin{array}{l}\text { Autoeficacia } \\
\text { Global }\end{array}$ & -.50 & -.49 & -.53 & -.32 & .41 \\
$\begin{array}{l}\text { Manejo del } \\
\text { dolor }\end{array}$ & & -.28 & -.31 & & .33 \\
$\begin{array}{l}\text { Actividades } \\
\text { físicas }\end{array}$ & -.48 & -.38 & -.50 & -.29 & .36 \\
$\begin{array}{l}\text { Manejo de } \\
\text { síntomas }\end{array}$ & -.55 & -.49 & -.42 & -.31 & .30 \\
Depresión & .65 & & & & -.40 \\
\hline
\end{tabular}

Nota: Solamente se han colocado los valores significativos $(p<.01)$

Al analizar las relaciones entre autoeficacia y estrategias de afrontamiento, se observa que todas las subescalas del cuestionario de autoeficacia se asocian de forma significativa y negativa con la estrategia de evitación y de forma significativa y positiva con la persistencia en la tarea. Estos datos podrían indicar, por una parte, que persistir en la tarea mejoraría la percepción de autoeficacia, sin embargo utilizar la evitación como estrategia de afrontamiento estaría relacionada con una disminución en la percepción de autoeficacia.
Con respecto a la estrategia de descanso, tres de las cuatro medidas de autoeficacia (global, actividad física y manejo de los síntomas) se han asociado negativamente con dicha estrategia de afrontamiento (Tabla 2). Esto podría estar indicando que, en estos pacientes, descansar no contribuye a mejorar su autoeficacia en el manejo de la fibromialgia.

Por último, señalar que de las dos respuestas emocionales evaluadas, la depresión ha correlacionado de forma negativa $(r=-.40)$ con la estrategia de afrontamiento de persistencia en la tarea (Tabla 2), es decir que a mayor persistencia en la actividad que se realice menor tendencia a padecer depresión.

\section{Discusión}

El objetivo de este estudio era analizar de forma descriptiva la relación entre considerarse capacitado para realizar diferentes y diversas actividades, es decir, las expectativas de autoeficacia, y la repercusión emocional y el afrontamiento del dolor en pacientes con fibromialgia.

En el caso de las variables emocionales evaluadas, ansiedad y depresión, si bien existe una relación significativa entre el padecimiento de ambos trastornos emocionales en nuestra muestra, cómo se ha demostrado en estudios previos (White, Nelson, Harth, Ostbye y Speechley, 2002), su presencia en la muestra es desigual. Así, mientras que el $44 \%$ de la muestra de nuestro estudio manifiesta un nivel de ansiedad clínico, sólo el $25 \%$ lo presenta en depresión. Los resultados presentados parecen corroborar la presencia de reacciones emocionales en la fibromialgia pero con un impacto diferente en los pacientes. Esto coincidiría con diferentes trabajos que han subrayado el carácter independiente de la relación entre ambas reacciones y la fibromialgia (Kurtzen, Gundersen y Svebak, 1998; Thieme, Turk, y Flor, 2004), señalándose incluso que el dolor generalizado parece asociarse de forma significativa con la ansiedad, mientras que la fatiga lo hace con la depresión (Kurtze y Svebak, 2001).

Respecto a los estilos de afrontamiento: protegerse (estrategia pasiva), descansar (estrategia pasiva) y las auto-instrucciones de afrontamiento (estrategia activa) y persistencia en la tarea (estrategia activa) son las más utilizadas por la muestra. Los resultados obtenidos coinciden parcialmente con diferentes trabajos realizados sobre este aspecto en fibromialgia, los cuales han subrayado el carácter pasivo del afrontamiento del dolor en este trastorno (Mellegard, Grossi y Soares, 2001; Soucase, Monsalve, Soriano y De Andrés, 2004). Sin embargo, la persistencia en la tarea, que es la estrategia más utilizada por los pacientes de esta muestra, correlaciona negativamente con la depresión. Esto podría estar indicando la conveniencia de dar un paso más allá y motivar a los pacientes a realizar estrategias más proactivas en la lucha contra los efectos negativos de la enfermedad que padecen. 
Los resultados obtenidos ponen de manifiesto que las expectativas de autoeficacia se relacionan de forma negativa con las reacciones emocionales de ansiedad y depresión y con las estrategias de afrontamiento de protección y descanso, mientras que se asocian de forma positiva con la persistencia en la tarea. Aunque no pueda establecerse una relación causal entre la autoeficacia percibida y el ajuste emocional y conductual en fibromialgia, los datos de este estudio suponen una prueba a favor tanto para continuar promocionando e incluyendo a las expectativas de autoeficacia como un objetivo más en cualquier tratamiento en fibromialgia, de acuerdo con los datos que ya avalan su uso terapéutico (Dolce, Crocker, Doley, 1986; Kores, Murphy, Rosenthal, Elias y North, 1990; Buckelew et al., 1996). En este sentido sería interesante transmitir, a la luz de los resultados obtenidos, a los pacientes de fibromialgia la importancia de la autoeficacia como estrategia para mejorar su situación. Así, tal y como señalaba Bandura (1992) las personas con confianza en sus capacidades, se enfrentan a las tareas difíciles como retos que han de ser superados y no como amenazas. Frente a la posibilidad de fracaso, aumentan y refirman aún más sus esfuerzos y se "crecen" ante los contratiempos. Las personas con un buen nivel de autoeficacia percibida se enfrentan a las situaciones de amenaza con la seguridad de que realmente son capaces de ejercer un control sobre ellas. En definitiva, parece que la autoeficacia genera estrategias de afrontamiento positivas ya que se tiende a no evitar situaciones y a persistir en las tareas. Con todo ello se podría contribuir a tener más nivel de actividad lo que redundaría en una mejor calidad de vida de estos pacientes (Gutiérrez y Gorricho, 2006).

Estos resultados permiten señalar algunas implicaciones para la práctica clínica. Por una parte, parece interesante que, al diseñar programas de intervención para este tipo de pacientes, se tuviera en cuenta la alta comorbilidad que en esta muestra se ha observado entre la ansiedad y la depresión. En segundo lugar podría ser de interés para los pacientes explicarles la importancia de su percepción de autoeficacia y como ésta puede ayudarles a sentirse mejor. Con respecto a las estrategias de afrontamiento, una cuestión a destacar sería la conveniencia de recomendar a estos pacientes que procuren no evitar situaciones o tareas y no centrarse excesivamente en el descanso; por el contrario, aconsejarles la perseverancia en sus tareas ya que todo ello, en su conjunto, les ayudará a percibirse más competentes en el manejo de su enfermedad.

Finalmente, señalar que la metodología descriptiva del estudio no permite la generalización y comprensión global de los mecanismos que explican la fibromialgia; en este sentido sería interesante realizar, con estas mismas variables, algún estudio de mediación o de modulación a través del que poder analizar dichos mecanismos. Por otra parte, y teniendo en cuenta las posibles implicaciones prácticas que se sugieren en este trabajo, sería conveniente que, en futuras investigaciones se consideraran los resultados antes señalados para el diseño de algún estudio experimental en el que se pusieran a prueba dichas sugerencias.

\section{Referencias}

Anderson, K. O., Dowds, B. N., Pelletz, R. E., Edwards, W. T. y Peeters-Asdourian, C. (1995). Development and initial validation of a scale to measure self-efficacy beliefs in patients with chronic pain. Pain, 63, 77-84.

Altman, A. (2004). The impact of self-efficacy, catastrophizing and pain intensity on psychological distress levels in individuals with chronic pain. Dissertation Abstracts International: Section B: The Science and Engineering, 65, 474.

Arnstein, P., Caudill, M., Mandle, C. L. Norris, A. y Beasley, R. (1999). Self efficacy as a mediator of the relationship between pain intensity, disability and depression in chronic pain patients. Pain, 80, 483-491.

Arnstein, P. (2000). The mediation of disability by self efficacy in different samples of chronic pain patients. Disability \& Rehabilitation, 22, 794-801.

Asghari, A. y Nicholas, M. K. (2001). Pain self-efficacy beliefs and pain behaviour. A prospective study. Pain, 94, 85-100.

Bandura, A. (1977). Self-efficacy: toward a unifying theory of behavioral change. Psychological Review, 84, 191-215.

Bandura, (1992). Discurso con motivo de su investidura como Doctor Honores Causa. xcSalamanca: Universidad de Salamanca.

Bayazit,Y., Gürsoy, S., Özer, E., Karakurum, G y Madenci, E. (2002). Neurotologic manifestations of fibromyalgic syndrome. Journal of Neurological Sciences, 196, 77-80.

Bennett, R.M. (2002). Rational management of fibromyalgia. Rheumatic Disease Clinics of North America, 28, 13-15

Bettina, S.A., Alpers, G.W., Süß, H., Friedel, E., Kosmützky, G., Geier, A. y Pauli, P. (2008). Affective pain modulation in fibromyalgia, somatoform pain disorder, back pain and health control. European Journal of Pain, 12, 329-338.

Brister, H., Turner, J. A., Aaron, L. A. y Mancl, L. (2006). Selfefficacy is associated with pain, functioning, and coping in patients with chronic temporomandibular disorder pain. Journal of Orofacial Pain, 20, 115-124.

Buckelew, S. P., Huyser, B., Hewett, J. E. y Parker, J. C., Johnson, J. C., Conway, R. y Kay, D. R. (1996). Self-efficacy predicting outcome among fibromyalgia subjects. Arthritis Care and Research, 9, 97-104.

Buckelew, S. P., Murray, S. E., Hewett, J. E., Johnson, J. y Huyser, B. (1995). Self efficacy, pain, and physical activity among fibromyalgia subjects. Arthritis Care and Research, 8, 43-50.

Buckelew, S. P., Parker, J. C., Keefe, F. J., Deuser, W. E., Crew, T. M., Conway, R., Kay, D. R. y Hewett, J. E. (1994). Selfefficacy and pain behavior among subjects with Fibromyalgia. Pain, 59, 377-384.

Buckhard, C.S. y Bjelle, A. (1996). Perceived control: a comparison of women with fibromyalgia, rheumatoid arthritis, and systemic lupus erythematosus using Swedish version of the Rheumatology Attitude Index. Scandinavian Journal of Rheumatology, 25, 300-306. 
Buescher, K. L., Johnston, J. A., Parker, J. C., Smarr, K. L., Bickelew, S. P., Anderson, S. K. y Walker, S. E. (1991). Relationship of self-efficacy to pain behaviour. Journal of Rheumatology, 18, 968-972.

Cooper-Evans, S., Alderman, N., Kinht, C. y Oddy, M. (2008). Self-esteem as predictor of psychological distress after severe acquired injury: an exploratory study. Neuropsychological Rehabilitation, 18, 607-626.

Dolce, J.J., Crocker, M. F. y Doleys, D.M. (1986). Prediction of outcome among chronic pain patients. Behaviour Research and Therapy, 24, 313-319.

Dresh, J., Polatin, P.B. y Gatchel, R.J. (2002). Chronic pain and psychopathology: Research findings and theoretical considerations. Psychosomatic Medicine, 64, 773-786.

Gutiérrez, R. y Gorricho, J. (2006). Fibromialgia. Boletín de información farmacoterapéutica de Navarra, 14, 17-26.

Han, S. S. y Lee, S. C. (2005). Effecting factors on depression in patients with fibromyalgia. Taehan Kanho Hakhoe Chi, 35, 87-94.

Jensen, M.P., Turner, J.A. y Romano, J.M. (1991). Self-efficacy and outcome expectancies: relationship to chronic pain coping strategies and adjustment. Pain, 44, 263-269.

Jensen, M.P., Turner, J.A., Romano, J.M. y Strom, S.E. (1995). The chronic pain coping inventory: development and preliminary validation. Pain, 60, 203-216.

Kerns, R.D., Rosenberg, R. y Jacob, M.C. (1994). Anger expression and chronic pain. Journal of Behavioral Medicine, 17, 57-67.

Kores, R.C., Murphy, W.D., Rosenthal, T.L., Elias, D.B. y North, W.C. (1990). Predicting outcome of chronic pain treatment via a modified self-efficacy scale. Behaviour Research and Therapy, 28, 165-169.

Kurtze, N., Gudersen, K.T., Svebak, S. (1998). The role of anxiety and depression in fatigue and patterns of pain among subgroups of fibromyalgia patients. The British Journal of Medical Psychology, 7, 185-194.

Kurtze, N. y Svebak, S. (2001). Fatigue and patterns of pain in fibromyalgia: correlations with anxiety, depression and comorbidity in a female country simple. The British Journal of Medical Psychology, 74, 523-537.

Lorig, K., Chastain, R.L., Ung, E., Shoor, S. y Holman, H.R. (1989). Development and evaluation of a scale to measure perceived self-efficacy in people with arthritis. Arthritis and Rheumatism, 32, 37-44.

McCracken, L.M., Zayfert, C. y Gross, R.T. (1992). The Pain Anxiety Symptoms Scale: development and validation of a scale to measure fear to pain. Pain, 50, 67-73.

Meeus, M. y Nijs, J. (2007). Central sensitization: a biopsychosocial explanation for chronic widespread pain in patients with fibromyalgia and chronic fatigue syndrome. Clinical Rheumatology, 26, 465-473.
Mellegard, M., Grossi, G., Soares, J.J. (2001). A comparative study of coping among women with fibromyalgia neck/ shoulder and back pain. International Journal of Behavioural Medicine, 8, 103-115.

Meredith, P., Strong, J. y Feeney, J.A. (2006). Adult attachment, anxiety, and pain self-efficacy as predictors of pain intensity and disability. Pain, 123, 146-154.

Okifuji, A., Turk, D.C. y Sherman, J.J. (2000). Evaluation of the relationship between depression and fibromyalgia syndrome: Why aren't all patients depressed? Journal of Rheumatology, 27, 212-219.

Oliver, K. y Cronan, T. A. (2005). Correlates of physical activity among women with Fibromyalgia Syndrome. Annals of Behavioral Medicine, 29, 44-53.

Rodríguez-Parra, M.J., Esteve, R. y López, A.E. (2000). Dolor crónico y estrategias de afrontamiento. Análisis y Modificación de Conducta, 27, 391-418.

Pérez-Pareja, J., Borrás, C., Palmer, A., Sesé, A., Molina, F. y Gonzalvo, J. (2004). Fibromialgia y emociones negativas. Psicothema, 16, 415-420.

Rahman, A., Ambler, G., Underwood, M.R. y Shipley, M.E. (2004). Important determinants of self-efficacy in patients with chronic pain musculoskeletal pain. Journal of Rheumatology, 31, 1187-1192.

Snow-Turek, A.L., Norris, M.P. y Tan, G. (1996). Active and passive coping strategies in chronic pain patients. Pain, 64, 455-462.

Soucase, B., Monsalve, V., Soriano, J. F. y de Andrés, J. (2004). Estrategias de afrontamiento ante el dolor y calidad de vida en pacientes diagnosticados de fibromialgia. Revista de la Sociedad Española de Dolor, 11, 353- 359.

Sung, K.W., Shin, I.H. y Lee, K.H. (2003). The factors influencing on depression of patients for fibromyalgia syndrome. Taehan Kanho Hakhoe Chi, 33, 609-617.

Thieme, K., Turk, D., y Flor, H. (2004). Comorbid depression and anxiety in fibromyalgia syndrome: relationship to somatic and psychosocial variables. Psychosomatic Medicine, 66, 837-844.

Tomey, T.C., Mann, J.D., Abashian, S. y Thompson-Pope, S. (1991). Relationship between perceived self-control of pain, pain description and functioning. Pain, 45, 129-133.

Turk, D.C., Okifuji, A. y Scharff, L. (1995). Chronic pain and depression: Role of perceived impact and perceived control in different age cohorts. Pain, 61, 93-101.

Weickgenant, A.L., Slater, M.A., Patterson, T.L., Atkinson, H., Grant, I. y Garfin, S.R. (1993). Coping activities in chronic low back pain: relationship with depression. Pain, 53, 95-103.

White, K.P., Nielson, W.R., Harth, M., Ostbye, T. y Speecheley, M. (2002). Chronic widespread musculoskeletal pain with or without fibromyalgia: psychological distress in a representative community adult sample. The Journal of Rheumatology, 29, 588-594. 
Wolfe, F.W., Smythe, H.A.., Yunas, M.B., Bennett, R.M. Bombardier, C., Goldenberg, D.L., Tugwell, P., Campbell, S.M., Abeles, M., Clark, P., Fam, G., Farber, S.J., Fiechtner, J.J., Franklin, C..M., Gatter, R.A., Hamaty, D., Lessard, J., Lichtbrou, A.S., Masl, A.T., MacCain, G.A., Reynolds, W.J., Romano, T.J., Russell, I.J. y Sheon, R.P. (1990). The American College of Rheumatology 1990, Criteria for the classification of fibrmoyalgia: Report of the Multicenter Criteria Committee. Arthritis and Rheumatism, 33, 160-172.
Zigmond, A. S. y Snaith, R. P. (1983). The Hospital Anxiety and Depression Scale. Acta Psychiatrica Scandinavica, 76, 361-370. 